\title{
DISPERSIVE ESTIMATES FOR TIME AND SPACE FRACTIONAL SCHRÖDINGER EQUATIONS
}

\author{
XIAOYAN SU ${ }^{1}$, SHILIANG ZHAO ${ }^{2}, \mathrm{MIAO} \mathrm{LI}^{3}$
}

\begin{abstract}
In this paper, we consider the Cauchy problem for the fractional Schrödinger equation $i D_{t}^{\alpha} u+(-\Delta)^{\frac{\beta}{2}} u=0$ with $0<\alpha<1, \beta>0$. We establish the dispersive estimates for the solutions. In particular, we prove that the decay rates are sharp.
\end{abstract}

2010 MSC: 26A33, 35Q41

Keywords: fractional Schrödinger equation, dispersive estimates

\section{INTRODUCTION}

Fractional differential equations have become a very important subject in the modern mathematical research because of its various applications in physics, biology, probability and so on. We refer the readers to [17] 14] 19] for a systematic introduction about fractional calculus. In probability, it has a close connection to the time-changed stochastic process, for more details, we refer the readers to [15]. In physics, fractional calculus are introduced to study the quantum phenomena, see 812 13. 21.

It is well known that the Schrödinger equation is one of the most important equations in the quantum mechanics, and the well-posedness of this equation has attracted the attention of thousands of researches, see [4] [5] 20, for more information. And its fractional generalisation can be divided into mainly three fields: (1) space fractional Schrödinger equation (SFSE), see 8, 12, 22, (2) time fractional Schrödinger equation (TFSE), see 1, 16, 23]; (3) and both time and space fractional Schrödinger equation (TSFSE), see [21, 24, 25].

Mathematical literatures about space fractional Schrödinger equation include [7, 10, 11] and references therein. From the physics viewpoint, (SFSE) can be derived by using the Feynman path integral techniques by replacing the Brownian quantum paths with Lévy stable paths, and the Markovian character are still remained, see [12] .

As for the time fractionalisation of the Schrödinger equation, the case is more controversial, mainly about if we should fractionalize the constant $i$. In [16, the author use Wick rotation to raise a fractional power of $i$, which turns out to be the clasical Schrödinger equations with a time dependent Hamiltonian. Whereas in the literature of $[1$ the authors derived the (TFSE) using the Feynman paths method, which doesn't change the constant $i$.

In this paper, we study the fractional Schrödinger equation in both time and space, which is a generalisation of the equation in [1]:

$$
\left\{\begin{array}{c}
i D_{t}^{\alpha} u(t, x)+(-\Delta)^{\frac{\beta}{2}} u(t, x)=0 \\
u(0, x)=\varphi(x)
\end{array}\right.
$$


where $0<\alpha<1, \beta>0, D_{t}^{\alpha}$ denotes the Caputo fractional derivative and $(-\Delta)^{\frac{\beta}{2}}$ denotes the fractional Laplacian.

The aim of this paper is to study the dispersive estimates of the equation (1.1). When $\alpha=1, \beta=$ 2, (1.1) becomes the classical Schrödinger equation. As is well known, the following estimates hold:

$$
\|u(t)\|_{L^{p^{\prime}\left(\mathbb{R}^{n}\right)}} \leq t^{-n\left(\frac{1}{p}-\frac{1}{2}\right)}\|u\|_{L^{p}\left(\mathbb{R}^{n}\right)} \quad \text { for } \quad 1 \leq p \leq 2, t>0
$$

The above decay estimates are very important in the nonlinear Schrödinger equation theory. See for example [5] and references therein. When $\alpha=1, \beta>0$ and $\beta \neq 1$, the dispersive estimates have been established in [6], and they have also shown that the decay rates are sharp.

In the first place, we get a $L^{\infty}$ estimates of the fundamental solutions of (1.1). In fact, the solution of (1.1) can be expressed as a Fourier multiplier,

$$
u(x, t)=\left[E_{\alpha}\left(-i t^{\alpha}|\xi|^{\beta}\right) \hat{\varphi}(\xi)\right]^{\vee}(x) \quad t>0,
$$

where $\varphi \in \mathcal{S}\left(\mathbb{R}^{n}\right)$ and $E_{\alpha}(z)$ is the Mittag-Leffler function. Since we have $\left|E_{\alpha}(z)\right| \leq C$ for $0<\alpha<$ $1, \frac{\pi}{2} \alpha<|\arg z|<\pi$ (see [18] ), it follows that $\|u(t)\|_{L^{2}\left(\mathbb{R}^{n}\right)} \leq C\|\varphi\|_{L^{2}\left(\mathbb{R}^{n}\right)}$.

Set $T_{t} \varphi(x)=\left[E_{\alpha}\left(-i t^{\alpha}|\xi|^{\beta}\right) \hat{\varphi}(\xi)\right]^{\vee}(x)=K_{t} * \varphi(x)$, where $K_{t}(x)=\left[E_{\alpha}\left(-i t^{\alpha}|\xi|^{\beta}\right)\right]^{\vee}(x)$ denotes the distributional kernel of $T_{t}$. Then we have the following result:

Proposition 1.1. For any fixed $t>0, K_{t}(\cdot) \in L^{\infty}\left(\mathbb{R}^{n}\right)$ iff $\beta>n$.

Note that when $\alpha=1, \beta>0$, similar results have been proved by [7]. Compared with the classical Schrödinger equation, where $\alpha=1, \beta=2$, and $K_{t}(x)=\frac{1}{(4 \pi i)^{\frac{n}{2}}} e^{\frac{i|x|^{2}}{4 t}} \in L^{\infty}\left(\mathbb{R}^{n}\right)$ for any fixed $t>0$, our result requires the condition that $\beta>n$ to make sure that $K_{t}(\cdot) \in L^{\infty}\left(\mathbb{R}^{n}\right)$, this phenomenon is caused by the different asymptotic behaviours of $E_{\alpha}\left(-i|\xi|^{2}\right)$ for $0<\alpha<1$ and $\alpha=1$. When $0<\alpha<1, E_{\alpha}\left(-i|\xi|^{2}\right)$ has quadratic decay, whereas when $\alpha=1, E_{\alpha}\left(-i|\xi|^{2}\right)=e^{-i|\xi|^{2}}$, which doesn't have any decay when $|\xi| \rightarrow \infty$ but oscillates.

As a result, when $\beta>n$, we can easily get the $L^{\infty}$ estimates for the solutions of (1.1) by Young's inequality. As for the case $0<\beta \leq n$, we need to localize the frequencies.

Let us consider a function $\phi \in C_{c}^{\infty}$ such that

$$
\phi(\xi)= \begin{cases}1, & \text { if } \quad|\xi| \leq 1 \\ 0, & \text { if } \quad|\xi| \geq 2\end{cases}
$$

and define the sequence $\left(\psi_{j}\right)_{j \in \mathbb{Z}} \subset \mathcal{S}\left(\mathbb{R}^{n}\right)$ by $\psi_{j}(\xi)=\phi\left(\frac{\xi}{2^{j}}\right)-\phi\left(\frac{\xi}{2^{j-1}}\right)$. We use $\psi$ to denote $\psi_{0}$. It is easy to notice that $\operatorname{supp} \psi_{j} \subset\left\{2^{j-1} \leq|\xi| \leq 2^{j+1}\right\}$. Set $P_{N} \varphi(x)=\left[\psi_{j}(\xi) \hat{\varphi}(\xi)\right]^{\vee}(x)$, where $N$ is dyadic number, i.e. $N=2^{j}$ for some integer $j$. The main results of this paper are as follows:

Theorem 1.2. (1) For $0<\alpha<1, \beta>0$ and dyadic number $N$, we have

$$
\left\|P_{N} T_{t} \varphi\right\|_{L^{\infty}\left(\mathbb{R}^{n}\right)} \lesssim \frac{N^{n}}{1+t^{\alpha} N^{\beta}}\|\varphi\|_{L^{1}\left(\mathbb{R}^{n}\right)}
$$

(2) For $0<\alpha<1, \beta>n$, we have

$$
\left\|T_{t} \varphi\right\|_{L^{\infty}\left(\mathbb{R}^{n}\right)} \lesssim t^{-\frac{n}{\beta} \alpha}\|\varphi\|_{L^{1}\left(\mathbb{R}^{n}\right)}
$$

Notice that when $0<\beta \leq n$, for fixed $N$, the time decay is $t^{-\alpha}$, which doesn't depend on the dimension. That is different from the classical case.

Besides, the above decay estimates are sharp in the following sense: 
Proposition 1.3. There exists $t_{0}, N_{0}$ such that for all $t>t_{0}, N>N_{0}$, we have

$$
\sup _{x \in \mathbb{R}^{n}}\left|K_{t}(x)\right| \gtrsim \frac{N^{n}}{1+t^{\alpha} N^{\beta}} .
$$

Moreover, we have the following dispersive estimates:

Theorem 1.4. For $0<\alpha<1,2 \leq r \leq \infty$, we have

$$
\begin{gathered}
\left\|T_{t} \varphi\right\|_{L^{\infty}\left(\mathbb{R}^{n}\right)} \lesssim\left(1+t^{\alpha}\right)^{-\left(1-\frac{2}{r}\right)}\left[\|\varphi\|_{\dot{B}_{r^{\prime}, 1}^{\frac{n}{r^{\prime}}}}+\|\varphi\|_{\dot{B}_{r^{\prime}, 1}^{\frac{n}{r^{\prime}-\beta\left(1-\frac{2}{r}\right)}}}\right] \\
\left\|T_{t} \varphi\right\|_{L^{r}\left(\mathbb{R}^{n}\right)} \lesssim\left(1+t^{\alpha}\right)^{-\left(1-\frac{2}{r}\right)}\left[\|\varphi\|_{\dot{B}_{r^{\prime}, 2}^{n\left(1-\frac{2}{r}\right)}}+\|\varphi\|_{\dot{B}_{r^{\prime}, 2}^{(n-\beta)\left(1-\frac{2}{r}\right)}\left(\mathbb{R}^{n}\right)}\right] .
\end{gathered}
$$

and

$$
\left\|T_{t} \varphi\right\|_{\dot{B}_{r, p}^{s}} \lesssim\left(1+t^{\alpha}\right)^{-\left(1-\frac{2}{r}\right)}\left[\|\varphi\|_{\dot{B}_{r^{\prime}, p}^{n\left(1-\frac{2}{r}\right)+s}}+\|\varphi\|_{\dot{B}_{r^{\prime}, p}^{(n-\beta)\left(1-\frac{2}{r}\right)+s}\left(\mathbb{R}^{n}\right)}\right] .
$$

Remark 1.5. The Strichartz estimates for the classical Schrödinger equation plays an important role in the well-posedness for the semilinear equations. But due to the loss of the semigroup property for the Mittag-Leffler functions, we can not use the $T T^{*}$ argument to prove the Strichartz estimates for the solutions of (1.1) directly.

Throughout this paper, we say $f \sim g$ if and only if there exists positive constants $c, C$ such that $c f \leq g \leq C f$. We say that $f \lesssim g$ if and only if there exists a positive constant $C$ such that $f \leq C g$.

\section{Preliminaries}

In this section, we give some explanations about the notations and introduce some basic knowledge about the Mittag-Leffler functions and Bessel functions and Besov spaces which will be used in the later sections.

Definition 2.1 (see [19] ). The fractional Riemann-Liouville integral of order $\alpha>0$ is defined as

$$
J^{\alpha} f(t)=\frac{1}{\Gamma(\alpha)} \int_{0}^{t}(t-s)^{\alpha-1} f(s) d s
$$

where

$$
g_{\alpha}(t)= \begin{cases}\frac{t^{\alpha-1}}{\Gamma(\alpha)} & \text { when } t>0 \\ 0 & \text { when } t \leq 0\end{cases}
$$

and $\Gamma(\alpha)$ is the Gamma function. Set moreover $g_{0}(t):=\delta(t)$, the Dirac delta-function. The fractional Caputo derivative of order $\alpha \in(0,1)$ is defined as

$$
\left(D_{t}^{\alpha} f\right)(t)=\int_{0}^{t} g_{1-\alpha}(t-s) f^{\prime}(s) d s=J^{1-\alpha} f^{\prime}(t) .
$$

We use the notation $\hat{f}, \check{f}$ to denote the Fourier and inverse Fourier transform of a function respectively, which is given by

$$
\hat{f}(\xi)=\frac{1}{(2 \pi)^{\frac{n}{2}}} \int_{\mathbb{R}^{n}} e^{-i x \cdot \xi} f(x) d x,
$$




$$
\check{f}(x)=\frac{1}{(2 \pi)^{\frac{n}{2}}} \int_{\mathbb{R}^{n}} e^{i x \cdot \xi} f(\xi) d \xi .
$$

Then the fractional Laplacian can be defined as

$$
(-\Delta)^{\frac{\beta}{2}} f(x)=\left[|\xi|^{\beta} \hat{f}(\xi)\right]^{\vee}(x) .
$$

2.1. Mittag-Leffler function. The Mittag-Leffler function is defined by the following power series:

$$
E_{\alpha}(z)=\sum_{k=0}^{\infty} \frac{z^{k}}{\Gamma(\alpha k+1)}, \quad \Re \alpha>0 .
$$

In this paper, we need the following asymptotic expansion of Mittag-Leffler function as $|z| \rightarrow \infty$ in various sectors of complex plan in the following proposition:

Proposition 2.2 (Proposition 3.6, [18] ). Let $0<\alpha<2$, and $\frac{\pi}{2} \alpha<\theta<\min \{\pi, \alpha \pi\}$, then we have the following asymptotic formula in which $k$ is an arbitrary positive integer:

$$
\begin{aligned}
& E_{\alpha}(z)=\frac{1}{\alpha} \exp \left(z^{\frac{1}{\alpha}}\right)-\sum_{j=1}^{k} \frac{z^{-j}}{\Gamma(1-\alpha j)}+O\left(|z|^{-1-k}\right), \quad|z| \rightarrow \infty, \quad|\arg z| \leq \theta, \\
& E_{\alpha}(z)=-\sum_{j=1}^{k} \frac{z^{-j}}{\Gamma(1-\alpha j)}+O\left(|z|^{-1-k}\right), \quad|z| \rightarrow \infty, \quad \theta \leq|\arg z| \leq \pi .
\end{aligned}
$$

2.2. Bessel function. [Appendix B, 9] ] For every $\nu \in \mathbb{C}$ such that $\Re v>-\frac{1}{2}$, the Bessel function of the first kind and of complex order $\nu$ can be defined by the Poisson representation formula:

$$
J_{\nu}(z)=\frac{1}{\Gamma\left(\frac{1}{2}\right) \Gamma\left(\nu+\frac{1}{2}\right)}\left(\frac{z}{2}\right)^{\nu} \int_{-1}^{1} e^{i z t}\left(1-t^{2}\right)^{\frac{2 \nu-1}{2}} d t .
$$

Another expression of $J_{\nu}$ as a power series for an arbitrary value if $\nu \in \mathbb{C}$ is provided by

$$
J_{\nu}(z)=\sum_{k=0}^{\infty}(-1)^{k} \frac{\left(\frac{z}{2}\right)^{\nu+2 k}}{\Gamma(k+1) \Gamma(k+\nu+1)}, \quad|z|<\infty, \quad|\arg z|<\pi .
$$

For the asymptotic behaviour of $J_{\nu}(z)$ as $z \rightarrow 0$ or $z \rightarrow \infty$, we have the following proposition.

Proposition 2.3 (Appendix B, 9] ). Let $\Re \nu>-\frac{1}{2}$, we have

(1) $J_{\nu}(z) \sim \frac{2^{-\nu} z^{\nu}}{\Gamma(\nu+1)}, \quad$ as $z \rightarrow 0$.

(2) $J_{\nu}(z)=\sqrt{\frac{2}{\pi z}} \cos \left(z-\frac{\pi \nu}{2}-\frac{\pi}{4}\right)+O\left(|z|^{-\frac{3}{2}}\right), \quad$ as $\quad|z| \rightarrow \infty, \quad-\pi+\delta<\arg z<\pi-\delta$.

In particular, we have $J_{-\frac{1}{2}}(z)=\sqrt{\frac{2}{\pi z}} \cos (z)$.

\subsection{Sobolev and Besov spaces. [Section 6.2, 2] ]}

Now we introduce the homogenous Sobolev spaces and Besov spaces. Let $s \in \mathbb{R}$ and $1 \leq p, q \leq \infty$, the homogenous Sobolev space is defined by

$$
\dot{H}^{s, p}\left(\mathbb{R}^{n}\right)=\left\{\varphi \in \mathcal{S}^{\prime}\left(\mathbb{R}^{n}\right):\|\varphi\|_{\dot{H}^{s, p}\left(\mathbb{R}^{n}\right)}<\infty\right\}
$$

where $\|\varphi\|_{\dot{H}^{s, p}\left(\mathbb{R}^{n}\right)}:=\left\|\sum_{j=-\infty}^{\infty}\left(|\xi|^{s} \psi_{j} \hat{\varphi}\right)^{\vee}\right\|_{L^{p}\left(\mathbb{R}^{n}\right)}$, and the homogenous Besov space is given by

$$
\dot{B}_{p, q}^{s}\left(\mathbb{R}^{n}\right)=\left\{\varphi \in \mathcal{S}^{\prime}\left(\mathbb{R}^{n}\right):\|\varphi\|_{\dot{B}_{p, q}^{s}\left(\mathbb{R}^{n}\right)}<\infty\right\}
$$


where

$$
\|\varphi\|_{\dot{B}_{p, q}^{s}}= \begin{cases}\left(\sum_{-\infty}^{\infty}\left(2^{j s q}\left\|\left(\psi_{j} \hat{\varphi}\right)^{\vee}\right\|_{L^{p}}\right)^{q}\right)^{1 / q}, & 1 \leq q<\infty \\ \sup _{j \in \mathbb{Z}} 2^{j s}\left\|\left(\psi_{j} \hat{\varphi}\right)^{\vee}\right\|_{L^{p}}, & q=\infty .\end{cases}
$$

Note that $\|\varphi\|_{\dot{H}^{s, p}\left(\mathbb{R}^{n}\right)}=0$ or $\|\varphi\|_{\dot{B}_{p, q}^{s}}=0$ if and only if $\operatorname{supp} \hat{\varphi}=\{0\}$, i.e. $u$ is a polynomial.

The nonhomogeneous Sobolev space is defined as

$$
H^{s, p}\left(\mathbb{R}^{n}\right)=\left\{\varphi \in \mathcal{S}^{\prime}\left(\mathbb{R}^{n}\right):\left[\left(1+|\xi|^{2}\right)^{\frac{s}{2}} \hat{\varphi}\right]^{\vee} \in L^{p}\left(\mathbb{R}^{n}\right)\right\},
$$

with the norm $\|\varphi\|_{H^{s, p}\left(\mathbb{R}^{n}\right)}:=\left\|\left[\left(1+|\xi|^{2}\right)^{\frac{\xi}{2}} \hat{\varphi}\right]^{\vee}\right\|_{L^{p}\left(\mathbb{R}^{n}\right)}$. The nonhomogeneous Besov space is given by

where

$$
B_{p, q}^{s}\left(\mathbb{R}^{n}\right)=\left\{\varphi \in \mathcal{S}^{\prime}\left(\mathbb{R}^{n}\right):\|\varphi\|_{B_{p, q}^{s}\left(\mathbb{R}^{n}\right)}<\infty\right\}
$$

$$
\|\varphi\|_{B_{p, q}^{s}}:=\left\|(\phi \hat{\varphi})^{\vee}\right\|_{L^{p}\left(\mathbb{R}^{n}\right)}+\left\{\begin{array}{ll}
\left(\sum_{j=1}^{\infty}\left(2^{j s}\left\|\left(\psi_{j} \hat{\varphi}\right)^{\vee}\right\|_{L^{p}}\right)^{q}\right)^{1 / q}, & 1 \leq q<\infty \\
\sup _{j \geq 1} 2^{j s}\left\|\left(\psi_{j} \hat{\varphi}\right)^{\vee}\right\|_{L^{p}}, & q=\infty .
\end{array} .\right.
$$

Notice that $L^{p}\left(\mathbb{R}^{n}\right)=\dot{B}_{2, p}^{0}\left(\mathbb{R}^{n}\right)$ and $\dot{B}_{p, q}^{s}\left(\mathbb{R}^{n}\right) \hookrightarrow \dot{B}_{q, p}^{s}\left(\mathbb{R}^{n}\right)$ if $q \leq p$.

\section{The PRoof of the MAIN Results}

Before proving our main results, we give the following two lemmas:

Lemma 3.1. For $0<\beta \leq n$,

(1) If $\beta \neq \frac{n}{m}$ for some positive integer $m$, then we have

$$
K_{t}(x)=\sum_{k=1}^{\left[\frac{n}{\beta}\right]} C_{k}|x|^{-n+\beta k} t^{-\alpha k}+C t^{-\frac{n}{\beta} \alpha} W\left(x t^{-\frac{\alpha}{\beta}}\right)
$$

where $W(x) \in L^{\infty}\left(\mathbb{R}^{n}\right)$.

(2) If $\beta=\frac{n}{m}$ for some positive integer $m$, we have

$$
K_{t}(x)=\sum_{k=1}^{m-1} C_{k}|x|^{-n+\beta k} t^{-\alpha k}+C_{m} t^{-m \alpha} W_{1}\left(x t^{-\frac{\alpha}{\beta}}\right)+C t^{-\frac{n}{\beta} \alpha} W\left(x t^{-\frac{\alpha}{\beta}}\right)
$$

where $W(x) \in L^{\infty}\left(\mathbb{R}^{n}\right)$ and $W_{1}(x) \sim \ln |x|$, as $|x| \rightarrow 0$.

In particular, we have $K_{t}(x) \notin L^{\infty}\left(\mathbb{R}^{n}\right)$ when $0<\beta \leq n$.

Proof. (1) To start with, we rewrite the kernel as:

$$
\begin{aligned}
K_{t}(x) & =\int_{\mathbb{R}^{n}} E_{\alpha}\left(-i t^{\alpha}|\xi|^{\beta}\right) e^{i x \cdot \xi} d \xi \\
& =t^{-\frac{n}{\beta} \alpha} \int_{\mathbb{R}^{n}} E_{\alpha}\left(-i|\xi|^{\beta}\right) e^{i x t^{-\frac{\alpha}{\beta}} \cdot \xi} d \xi .
\end{aligned}
$$

By writing $\eta=x t^{-\frac{\alpha}{\beta}}$, we have

$$
\begin{aligned}
K_{t}(x) & =t^{-\frac{n}{\beta} \alpha} \int_{\mathbb{R}^{n}} E_{\alpha}\left(-i|\xi|^{\beta}\right) e^{i \eta \cdot \xi} d \xi \\
& =t^{-\frac{n}{\beta} \alpha} \int_{\mathbb{R}^{n}} E_{\alpha}\left(-i|\xi|^{\beta}\right) \Phi(\xi) e^{i \eta \cdot \xi} d \xi+t^{-\frac{n}{\beta} \alpha} \int_{\mathbb{R}^{n}} E_{\alpha}\left(-i|\xi|^{\beta}\right)(1-\Phi(\xi)) e^{i \eta \cdot \xi} d \xi,
\end{aligned}
$$


where $\Phi \in C^{\infty}\left(\mathbb{R}^{n}\right)$ with

$$
\Phi(s)= \begin{cases}1 & |s| \geq 2 \\ 0 & |s| \leq 1\end{cases}
$$

Now, we define $K_{t}^{1}(\eta):=\int_{\mathbb{R}^{n}} E_{\alpha}\left(-i|\xi|^{\beta}\right) \Phi(\xi) e^{i \eta \cdot \xi} d \xi$ and $K_{t}^{2}(\eta):=\int_{\mathbb{R}^{n}} E_{\alpha}\left(-i|\xi|^{\beta}\right)(1-\Phi(\xi)) e^{i \eta \cdot \xi} d \xi$. One directly see that

$$
\left|K_{t}^{2}(\eta)\right| \leq C \quad \text { for } \quad t>0, \eta \in \mathbb{R}^{n} .
$$

For the first term, we use the Taylor expansion of the Mittag-Leffler function to see that

$$
K_{t}^{1}(\eta)=\int_{\mathbb{R}^{n}}\left(\sum_{k=1}^{\left[\frac{n}{\beta}\right]} C_{k}|\xi|^{-\beta k}+R(\xi)\right) \Phi(\xi) e^{i \eta \cdot \xi} d \xi,
$$

where $R(\xi))=O\left(|\xi|^{-\beta\left[\frac{n}{\beta}\right]}\right)$ as $|\xi| \rightarrow \infty$. Using the identity

$$
\mathcal{F}^{-1}\left(|\xi|^{-\theta}\right)=C|x|^{-n+\theta}, \quad \text { for } \quad 0<\theta<n,
$$

we see that

$$
\begin{aligned}
K_{t}^{1}(\eta) & =\sum_{k=1}^{\left[\frac{n}{\beta}\right]} C_{k}^{\prime}|\eta|^{-n+\beta k}+\int_{\mathbb{R}^{n}} \sum_{k=1}^{\left[\frac{n}{\beta}\right]} C_{k}|\xi|^{-\beta k}(\Phi(\xi)-1) e^{i \eta \cdot \xi} d \xi+\tilde{W}(\eta) \\
& =\sum_{k=1}^{\left[\frac{n}{\beta}\right]} C_{k}^{\prime}|\eta|^{-n+\beta k}+W(\eta),
\end{aligned}
$$

where $W(\eta) \in L^{\infty}\left(\mathbb{R}^{n}\right)$.

(2) Note that it is sufficient to show that

$$
W_{1}(\eta)=\int_{\mathbb{R}^{n}}|\xi|^{-n} \Phi(\xi) e^{i \eta \cdot \xi} d \xi \sim \ln (|\eta|) \quad \text { as } \quad|\eta| \rightarrow 0 .
$$

In fact, we have

Note that

$$
W_{1}(\eta)=\int_{|\xi| \geq 2}|\xi|^{-n} e^{i \eta \cdot \xi} d \xi+\int_{1 \leq|\xi| \leq 2}|\xi|^{-n} \Phi(\xi) e^{i \eta \cdot \xi} d \xi
$$

as for the first term,

$$
\int_{1 \leq|\xi| \leq 2}|\xi|^{-n} \Phi(\xi) e^{i \eta \cdot \xi} d \xi \leq C \quad \text { for } \quad \forall \eta \in \mathbb{R}^{n}
$$

$$
\begin{aligned}
\int_{|\xi| \geq 2}|\xi|^{-n} e^{i \eta \cdot \xi} d \xi & =\int_{2}^{+\infty} r^{-1}(r|\eta|)^{\frac{2-n}{2}} J_{\frac{n-2}{2}}(r|\eta|) d r \\
& =\int_{2|\eta|}^{+\infty} t^{-1} t^{\frac{2-n}{2}} J_{\frac{n-2}{2}}(t) d t \\
& =\int_{2|\eta|}^{1} t^{-1} t^{\frac{2-n}{2}} J_{\frac{n-2}{2}}(t) d t+\int_{1}^{+\infty} t^{-1} t^{\frac{2-n}{2}} J_{\frac{n-2}{2}}(t) d t
\end{aligned}
$$

The result now follows because

$$
\left|\int_{1}^{+\infty} t^{-1} t^{\frac{2-n}{2}} J_{\frac{n-2}{2}}(t) d t\right|<\infty
$$


and

$$
\int_{2|\eta|}^{1} t^{-1} t^{\frac{2-n}{2}} J_{\frac{n-2}{2}}(t) d t \sim \int_{2|\eta|}^{1} \frac{d t}{t} \sim \ln |\eta| .
$$

Here we used the property of Bessel functions that

$$
J_{\frac{n-2}{2}}(t) \sim \frac{1}{\Gamma\left(\frac{n}{2}\right)}\left(\frac{t}{2}\right)^{\frac{n-2}{2}} .
$$

This concludes the proof of the lemma.

Remark 3.2. When we compare the above results with the case $\alpha=1, \beta=2$, the asymptotic expansion of equation (3.10) isn't valid. In that case, $E_{\alpha}\left(-i|\xi|^{2}\right)=e^{-i|\xi|^{2}}$, thus $K_{t}^{1}(\eta) \in L^{\infty}(\eta)$.

Now we are ready to prove Theorem 1.2 .

Proof. To start with, we prove second statement is valid. First we recall that, for any $\frac{\pi}{2} \alpha<$ $|\arg z| \leq \pi$

Then for $\beta>n$, we have

$$
\left|E_{\alpha}(z)\right| \lesssim \begin{cases}C_{R} & |z| \leq R \\ |z|^{-1} & |z|>R\end{cases}
$$

$$
\begin{aligned}
\left|K_{t}(x)\right| & =t^{-\frac{n}{\beta} \alpha}\left|\int_{\mathbb{R}^{n}} E_{\alpha}\left(-i|\xi|^{\beta}\right) e^{i \eta \cdot \xi} d \xi\right| \\
& \leq t^{-\frac{n}{\beta} \alpha} \int_{\mathbb{R}^{n}}\left|E_{\alpha}\left(-i|\xi|^{\beta}\right)\right| d \xi \\
& \lesssim t^{-\frac{n}{\beta} \alpha} \int_{\mathbb{R}^{n}} \frac{d \xi}{1+|\xi| \beta} \lesssim t^{-\frac{n}{\beta} \alpha} .
\end{aligned}
$$

As for the case $0<\beta \leq n$, and $N$ is a dyadic number, i.e. $N=2^{j}$

$$
\begin{aligned}
\left|K_{t}^{N}(x)\right| & =\int_{\mathbb{R}^{n}} E_{\alpha}\left(-i t^{\alpha}|\xi|^{\beta}\right) \psi\left(\frac{|\xi|}{N}\right) e^{i x \cdot \xi} d \xi \\
& =N^{n} \int_{\mathbb{R}^{n}} E_{\alpha}\left(-i t^{\alpha} N^{\beta}|\xi|^{\beta}\right) \psi(\xi) e^{i N x \cdot \xi} d \xi \\
& =N^{n} \int_{0}^{\infty} E_{\alpha}\left(-i t^{\alpha} N^{\beta}|r|^{\beta}\right) \psi(r) r^{n-1} J_{\frac{n-2}{2}}(r N|x|)(N|x|)^{\frac{2-n}{2}} d r,
\end{aligned}
$$

since $\operatorname{supp} \psi \subseteq \mathcal{C}=\left\{|\xi|: \frac{1}{2} \leq|\xi| \leq 2\right\}$, we have

$$
\left|K_{t}^{N}(x)\right| \lesssim N^{n} \int_{\frac{1}{2}}^{2}\left|E_{\alpha}\left(-i t^{\alpha} N^{\beta} r^{\beta}\right)\right| d r \lesssim \frac{N^{n}}{1+t^{\alpha} N^{\beta}},
$$

Hence, by Young's inequality for convolutions, we have proven the theorem.

Notice that, when $0<\beta \leq n$, according to our method, the decay rate with respect to time is $t^{-\alpha}$, which doesn't depend on the dimension.

As results of the theorems, we have the following two corollaries: 
Corollary 3.3. Let $\alpha>0, \beta>n$. If $p \in[2, \infty)$ and $t>0$, then $T_{t}$ maps $L^{p^{\prime}}\left(\mathbb{R}^{n}\right)$ continuously to $L^{p}\left(\mathbb{R}^{n}\right)$ and we have the estimates

$$
\left\|T_{t} \varphi\right\|_{L^{p}\left(\mathbb{R}^{n}\right)} \lesssim t^{-\frac{2 n \alpha}{\beta}\left(\frac{1}{2}-\frac{1}{p}\right)}\|\varphi\|_{L^{p^{\prime}}\left(\mathbb{R}^{n}\right)}, \quad \text { for all } \quad \varphi \in L^{p^{\prime}}\left(\mathbb{R}^{n}\right) .
$$

Proof. Using Riesz-Thorin interpolation theorem, we immediately get the result.

Corollary 3.4. Let $\alpha>0, \beta>n$, for $t>0$ then we have

$$
\left\|T_{t} \varphi\right\|_{\dot{H}^{s, p}\left(\mathbb{R}^{n}\right)} \lesssim t^{-\frac{2 n \alpha}{\beta}\left(\frac{1}{2}-\frac{1}{p}\right)}\|\varphi\|_{\dot{H}^{s, p}\left(\mathbb{R}^{n}\right)}, \quad \text { for all } \quad \varphi \in \dot{H}^{s, p^{\prime}}\left(\mathbb{R}^{n}\right)
$$

and

$$
\left\|T_{t} \varphi\right\|_{\dot{B}_{p, q}^{s}\left(\mathbb{R}^{n}\right)} \lesssim t^{-\frac{2 n \alpha}{\beta}\left(\frac{1}{2}-\frac{1}{p}\right)}\|\varphi\|_{\dot{B}_{p^{\prime}, q}^{s}\left(\mathbb{R}^{n}\right)}, \quad \text { for all } \quad \varphi \in \dot{B}_{p, q}^{s}\left(\mathbb{R}^{n}\right)
$$

where $s \in \mathbb{R}$ and $2 \leq p \leq \infty$ and $1 \leq q \leq \infty$. And the above estimates holds for the nonhomgeneous Sobolev spaces and Besov spaces.

In the above theorems, we give the upper bound for the fundamental solution of the equation (1.1), now we prove that the estimate in the Theorem 1.2 is optimal.

Proof. Note that we can always choose $t, N$ such that $t^{\alpha} N^{\beta} \geq 1$, so $\frac{N^{n}}{1+t^{\alpha} N^{\beta}} \sim t^{-\alpha} N^{n-\beta}$. Therefore, it is sufficient to show that exists $t_{0}, N_{0}$ such that

$$
\sup _{x \in \mathbb{R}^{n}}\left|K_{t}(x)\right| \gtrsim t^{-\alpha} N^{n-\beta} \quad \text { for } \quad t>t_{0}, \quad N \geq N_{0} .
$$

Notice that

$$
\left|K_{t}(x)\right|=N^{n}\left|\int_{\frac{1}{2}}^{2} E_{\alpha}\left(-i t^{\alpha} N^{\beta} r^{\beta}\right) \psi(r) r^{n-1}(r|x|)^{\frac{2-n}{2}} J_{\frac{n-2}{2}}(r|x|) d r\right|:=N^{n}|I|,
$$

we write $I$ as the following,

$$
\begin{aligned}
I= & \int_{\frac{1}{2}}^{2}\left(E_{\alpha}\left(-i t^{\alpha} N^{\beta} r^{\beta}\right)-i t^{-\alpha} N^{-\beta} r^{-\beta}\right) \psi(r) r^{n-1}(r|x|)^{\frac{2-n}{2}} J_{\frac{n-2}{2}}(r|x|) d r \\
& +\int_{\frac{1}{2}}^{2} i t^{-\alpha} N^{-\beta} r^{-\beta} \psi(r) r^{n-1}(r|x|)^{\frac{2-n}{2}} J_{\frac{n-2}{2}}(r|x|) d r \\
:= & I_{1}+I_{2},
\end{aligned}
$$

then we have $\left|K_{t}(x)\right| \gtrsim N^{n}\left(\left|I_{1}\right|-\left|I_{2}\right|\right)$.

It is easy to check that

$$
\left|I_{1}\right| \lesssim\left(t^{\alpha} N^{\beta}\right)^{-2} \sim t^{-\alpha} N^{-\beta}\left(t^{\alpha} N^{\beta}\right)^{-1}
$$

For the second term, notice that

$$
\left|I_{2}\right| \sim N^{-\beta} t^{-\alpha}\left|\int_{\frac{1}{2}}^{2} \psi(r) r^{n-1-\beta}(r N|x|)^{\frac{2-n}{2}} J_{\frac{n-2}{2}}(r N|x|) d r\right|,
$$

and we can always choose $x$ such that $2 N|x|$ is small enough such that $J_{\frac{n-2}{2}}(r N|x|)>0$ is valid for all $\frac{1}{2}<r<2$. Therefore, we have proven that $\left|K_{t}(x)\right| \gtrsim N^{n-\beta} t^{-\alpha}$.

Now we give the proof for the Theorem 1.4 
Proof. (1) To start with, we define $P_{\sim N}=\sum_{\frac{N}{2} \leq 2^{j} \leq 2 N} P_{2^{j}}$, notice that $\psi\left(\frac{\xi}{2 N}\right)+\psi\left(\frac{\xi}{N}\right)+\psi\left(\frac{2 \xi}{N}\right)=$ $\phi\left(\frac{\xi}{2^{j+1}}\right)-\phi\left(\frac{\xi}{2^{j-2}}\right)$, which equals 1 on the support of $\psi$, then we have

$$
\begin{aligned}
P_{N}\left(T_{t} \varphi\right)(x) & =\int_{\mathbb{R}^{n}} e^{i x \xi} E_{\alpha}\left(-i t^{\alpha}|\xi|^{\beta}\right) \psi\left(\frac{\xi}{N}\right) \hat{\varphi}(\xi) d \xi \\
& =\int_{\mathbb{R}^{n}} e^{i x \xi} E_{\alpha}\left(-i t^{\alpha}|\xi|^{\beta}\right) \psi\left(\frac{\xi}{N}\right)\left(\psi\left(\frac{\xi}{2 N}\right)+\psi\left(\frac{\xi}{N}\right)+\psi\left(\frac{2 \xi}{N}\right)\right) \hat{\varphi}(\xi) d \xi
\end{aligned}
$$

By Hölder inequality and the fact that $\left|E_{\alpha}(z)\right| \leq C$ for $\frac{\pi}{2} \alpha<|\arg z| \leq \pi$, we obtain that

$$
\begin{aligned}
\left|P_{N}\left(T_{t} \varphi\right)(x)\right| & \leq\left\|\widehat{P_{\sim N} \varphi}\right\|_{L^{r}}\left\|E_{\alpha}\left(-i t^{\alpha}|\xi|^{\beta}\right) \psi\left(\frac{\xi}{N}\right)\right\|_{L^{r^{\prime}}} \\
& \lesssim N^{\frac{n}{r^{\prime}}}\left\|\widehat{P_{\sim N} \varphi}\right\|_{L^{r}} .
\end{aligned}
$$

By the Hausdorff-Young inequality, we have for $t>0$,

$$
\left\|P_{N}\left(T_{t} \varphi\right)\right\|_{L^{\infty}} \lesssim N^{\frac{n}{r^{\prime}}}\left\|P_{\sim N} \varphi\right\|_{L^{r^{\prime}}}
$$

In particular, we have

$$
\left\|P_{N}\left(T_{t} \varphi\right)\right\|_{L^{\infty}} \lesssim N^{\frac{n}{2}}\left\|P_{\sim N} \varphi\right\|_{L^{2}} .
$$

Taking the sum in $N$ in inequality of (3.2), we get

$$
\left\|T_{t} \varphi\right\|_{L^{\infty}} \lesssim\|\varphi\|_{\dot{B}_{r^{\prime}, 1}^{\frac{n}{r^{\prime}}}}
$$

Notice that $P_{N}\left(T_{t} \varphi\right)=K_{N} * P_{\sim N} \varphi$, and according to Theorem 1.2, we have

$$
\left\|P_{N}\left(T_{t} \varphi\right)\right\|_{L^{\infty}} \lesssim t^{-\alpha} N^{n-\beta}\left\|P_{\sim N} \varphi\right\|_{L^{1}} .
$$

Thus, by interpolation for $2 \leq r \leq \infty$ between (3.2) and (3.5), we have for $t>0$,

$$
\left\|T_{t} \varphi\right\|_{L^{\infty}} \lesssim t^{-\alpha\left(1-\frac{2}{r}\right)} N^{\frac{n}{r^{\prime}}-\beta\left(1-\frac{2}{r}\right)}\left\|P_{\sim N} \varphi\right\|_{L^{r^{\prime}}},
$$

Taking sum in $N$, we have the following result,

$$
\left\|T_{t} \varphi\right\|_{L^{\infty}} \lesssim t^{-\alpha\left(1-\frac{2}{r}\right)}\|\varphi\|_{\dot{B}_{r^{\prime}, 1}^{\frac{n}{r^{\prime}}-\beta\left(1-\frac{2}{r}\right)}\left(\mathbb{R}^{n}\right)} .
$$

Combine (3.4) and (3.6), we get the inequality (1.2).

On the other hand, we have

$$
\left\|P_{N}\left(T_{t} \varphi\right)\right\|_{L^{\infty}} \lesssim N^{n}\left\|P_{\sim N} \varphi\right\|_{L^{1}}
$$

and the $L^{2}$ estimates:

$$
\left\|P_{N}\left(T_{t} \varphi\right)\right\|_{L^{2}} \lesssim\left\|P_{\sim N} \varphi\right\|_{L^{2}} .
$$

Combining the inequalities of (3.8) and (3.9), we have

$$
\left\|P_{N}\left(T_{t} \varphi\right)\right\|_{L^{r}} \lesssim N^{n\left(1-\frac{2}{r}\right)}\left\|P_{\sim N} \varphi\right\|_{L^{r^{\prime}}}
$$

and summing in $N$, we have

$$
\left\|T_{t} \varphi\right\|_{L^{r}\left(\mathbb{R}^{n}\right)} \lesssim\|\varphi\|_{\dot{B}_{r^{\prime}, 2}^{n\left(1-\frac{2}{r}\right)}}
$$


And, by interpolating between (3.5) and (3.10), we have

$$
\left\|P_{N}\left(T_{t} \varphi\right)\right\|_{L^{r}} \lesssim t^{-\alpha\left(1-\frac{2}{r}\right)} N^{(n-\beta)\left(1-\frac{2}{r}\right)}\left\|P_{\sim N} \varphi\right\|_{L^{r^{\prime}}}
$$

Hence, summation in $N$ gives the result of (1.3).

Similarly, if we combine (3.10) and (3.9), we proved our last inequality of (1.4).

\section{REFERENCES}

[1] B. N. Narahari Achar, B. T. Yale, and J. W. Hanneken, Time fractional Schrödinger equation revisited, Adv. Math. Phys., 2013(2013), 1-11.

[2] J. Bergh and J. Löfström, "Interpolation Spaces," Springer-Verlag, New-York, 1976.

[3] T. Boulenger, D. Himmelsbach, and E. Lenzamann, Blowup for fractional NLS, J. Funct. Anal., 271 (2016), 2569-2603.

[4] J. Bourgain, "Global Solutions of Nonlinear Schrödinger Equations", AMS, 1999.

[5] T. Cazenave, "Semilinear Schrödinger Equations", vol. 10, New York University, Courant Institute of Mathematical Sciences, Ney York; American Mathematical Society, Providence, RI, 2003.

[6] Y. Cho, H. Ozawa, and S. Xia, Remarks on some dispersive estimates, Commun. Pur. App. Anal., 10 (2011), 1121-1128.

[7] V. Duong Dinh, On the blowup solutions to the focusing $L^{2}$-supercritical nonlinear fractional Schrödinger equations, arXiv:1802.0289 (2018).

[8] N. Laskin, Fractional quantum mechanics and Lévy path integrals, Phys. Lett. A, 268 (2000), 298-305.

[9] L. Grafakos, "Classical Fourier Analysis", Graduate Texts in Mathematics, Spring New York, 2008.

[10] Y. Hong and Y. Sire, On fractional Schrödinger equations in Sobolev spaces, Commun. Pur. App. Anal., 14(2015), 2265-2282.

[11] A. Ionescu and F. Pusateri, Nonlinear fractional Schrödinger equations in one dimension, J. Funct. Anal., 266(2014), 139-176.

[12] N. Laskin, Fractional Schrödinger equation, Phys. Rev. E, 66(2002), 56-108.

[13] N. Laskin, Time fractional quantum mechanics, Chaos, solitons and Fractals, 102(2017), 16-28.

[14] F. Mainard, "Fractional Calculus and Waves in Linear Viscoelasticity: an introduction to mathematical models", World Scientific, 2010.

[15] M. M. Meerschaert and A. Sikorskii, "Stochastic Models for Fractional Calculus," Vol. 43, De Gruyter Studies in Mathematics, Walter de Gruyter, Berlin/ Boston, 2012.

[16] M. Naber, Time fractional Schrödinger equation, J. Math. Phys., 45(2004), 3339-3352.

[17] I. Podlubny, "Fractional Differential Equations," Mathematics in Science and Engineering, 1999.

[18] R. Gorenflo, A. A. Kilbas, F. Mainarid, S. V. Rogosin, "Mittag-Leffler Functions, Related Topics and Applications," Springer Monographs in Mathematics, Springer-Verlag Berlin Heidelberg, 2014.

[19] O. I. Marichev, S. G. Samko, A. A. Kilbas, "Fractional Derivative and Integral," Gordon and Breach Science, 1993.

[20] T. Tao, "Nonlinear Dispersive Equations: Local and Global Analysis," Regional Conference Series in Mathematics, number 106, 2006.

[21] N. Laskin, "Fractional Quantum Mechanics," World Scientific, 2018.

[22] N. Laskin, Fractional quantum mechanics, Phys. Rev. E, 62(2000), 3135.

[23] S. S. Bayin, Time fractional Schrödinger equation: Fox's H-functions and the effective potential, J. Math. Phys. 54 (2003), 012103.

[24] S. Wang and M. Xu, Generalised fractional Schrödinger equation with space-time derivatives, J. Math. Phys.48(2007), 043502.

[25] J. Dong and M. Xu, Space-time fractional Schrödinger equation with time dependent potentials, J. Math. Anal. Appl., 344, 1005.

${ }^{1}$ Institute of Applied Physics and Computational Mathematics, 100094, P.R.China.

E-mail: 245440714@qq.com

2 Department of Mathematics, Sichuan University, 610064, P.R.China.

E-mail: zhaoshiliang@scu.edu.cn 
${ }^{3}$ Department of Mathematics, Sichuan University, 610064, P.R.China.

E-mail: mli@scu.edu.cn 